\title{
Vortex domain wall chirality rectification due to the interaction with end domain spin structures in permalloy nanowires
}

\author{
E.-S. Wilhelm, ${ }^{1,2}$ D. McGrouther ${ }^{2}$ L. Heyne, ${ }^{1}$ A. Bisig, ${ }^{1}$ and M. Kläui ${ }^{1, a)}$ \\ Fachbereich Physik, Universität Konstanz, Universitätsstr. 10, 78457 Konstanz, Germany \\ ${ }^{2}$ Department of Physics and Astronomy, University of Glasgow, Scotland G12 $8 Q Q$, United Kingdom
}

\begin{abstract}
The interaction of vortex domain walls with the end domain spin structure present at the rectangular end of a ferromagnetic nanowire is investigated using Lorentz transmission electron microscopy. When vortex walls are moved with short field pulses towards the wire end an end vortex is formed, whose chirality is independent of the original vortex wall chirality but is determined by the spin configuration of the end domain. This acts as a domain wall chirality "rectifier," which could be useful for applications based on domain walls. The observed chirality transformations are reproduced by micromagnetic simulations showing a complex reversal mechanism.
\end{abstract}

Domain walls and their controlled manipulation in nanowires $^{1-4}$ have potential for data storage $\mathrm{I}^{\mathrm{I}}$ and logic devices. ${ }^{2}$ Typically domain walls in soft magnetic nanowires can be classified into transverse or vortex walls depending on their spin structure [see Fig. 1, top wire: $\wedge$-shaped kink transverse walls, other: vortex wall with clockwise chirality (inplane circulation direction of the magnetization)]..$^{4-7}$

For pinning at artificial pinning sites such as notches, the detailed spin structure, such as the chirality of the vortex domain wall is the key factor. ${ }^{4,8}$ The potential landscape around the pinning site strongly depends on the wall chirality. ${ }^{4,9-11}$ The generated walls however have mostly random chirality, which leads to badly controlled pinning. The controlled switching of chirality has received little attention so far, even though it is essential for application in a device where reliable pinning is required. One method to obtain reliable pinning may be through the use of a chirality rectifier that sets the vortex wall chirality to a predetermined sense (clockwise or counter-clockwise).

In this Letter, we study domain wall depinning, transformations, and interactions of the domain wall with the end domain under applied magnetic field pulses using Lorentz transmission electron microscopy. When a vortex wall arrives at the wire end, we observe that the sense of rotation of the generated end vortex is independent of the incoming vortex wall chirality and set by the end domain spin structure meaning that this acts as a chirality rectifier.

The permalloy $\left(\mathrm{Ni}_{80} \mathrm{Fe}_{20}\right)$ wires studied were $500 \mathrm{~nm}$ wide and $20 \mathrm{~nm}$ thick and fabricated by focused ion beam milling on $\mathrm{Si}_{3} \mathrm{~N}_{4}$ membranes. Two sets of three different types of wires were produced [Fig. 1]. One was a conventional zigzag pattern with an angle of $132^{\circ}$ (geometry 1), the other types had a section of a ring instead of a sharp corner with a mean curvature of $2.23 \mu \mathrm{m}$ (geometry 2) and $3.23 \mu \mathrm{m}$ (geometry 3).

In order to nucleate domain walls a magnetic field parallel to the electron beam is applied by the objective lens of the transmission electron microscope (TEM) [see Fig. 1]. At

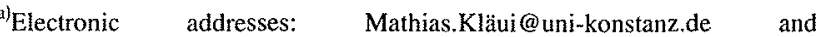
mathias@klaeui.de. Also at: Zukunftskolleg, Universtiät Konstanz, 78457 Konstanz, Germany.
}

zero-field the magnetic moments relax and align parallel to the wire edges and a domain wall is nucleated at each bend. ${ }^{12}$ To study the magnetization switching, magnetic field pulses of varying height and $2 \mu$ s length are applied in the direction of the mean wire axis. The pulses are triggered manually and each pulse is followed by the observation of the spin configuration. The field is applied using two small gold wires in series next to the sample. ${ }^{3}$ We then image the spin structure by Lorentz microscopy. We employ Fresnel mode where regions of increased and decreased intensity, appearing as white and black contrast, occur at the position of the domain walls. ${ }^{14}$ The technique is sensitive to the gradient of magnetization. The resulting contrast of the nucleated domain walls is seen together with a sketch in Fig. 1 (see Ref. 4 for more details). Black contrast means that magnetic moments turn to the right, white contrast means that magnetic moments turn to the left, and gray areas contain uniform magnetization. If

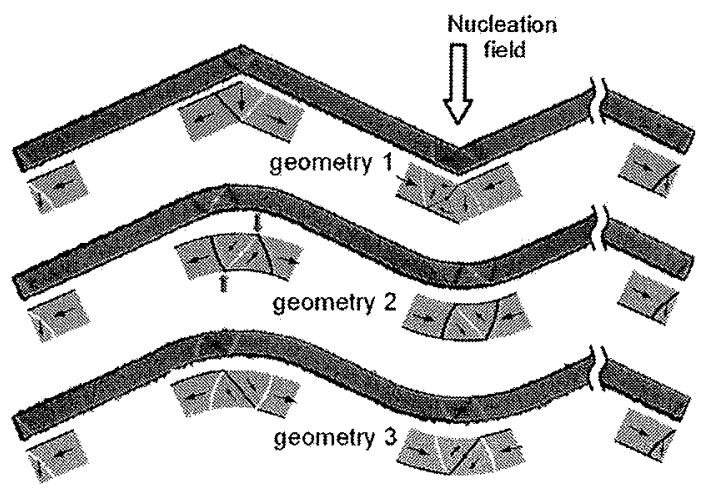

FIG. 1. (Color online) Three different geometries after the domain wall nucleation process with 150 Oe magnetic field component in the sample plane perpendicular to the wires (indicated by the arrow). Tail-to-tail ( $2 t$ ) domain walls are seen at the $\wedge$-shaped bend and head-to-head (h2h) walls at $\checkmark$-bend. Schematic drawings visualize the approximate spin structure of the walls (note that the arrows represent the average magnetization in a large area). In geometry 1, a transverse domain wall is seen at the left kink and a vortex domain wall with clockwise (cw) chirality one further to the right. In geometry 2 all vortex walls have ccw chirality. The topological edge defects of a vortex domain wall, that are connected by a diagonal line, are indicated by arrows in the sketch of the left wall. There are two $\mathrm{cw}$ vortex domain walls in geometry 3 . In all wires a white end domain wall is visible at the left and a black end domain wall at the right end. 


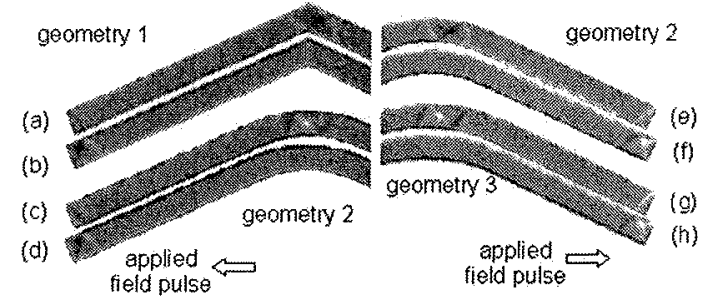

FIG. 2. After reversal of the nucleation field compared to Fig. 1: Initial h2h domain walls at the bends, black end domain walls at the left end [(a) and (c)] and white end domain walls at the right end [(e) and (g)]. Left end: (a) cw vortex wall, after a field pulse of 310 forms end vortex wall with same chirality (b). (c) ccw vortex wall that transforms to $\mathrm{cw}$ end yortex wall after a field pulse of 6 Oe (d). Right end: (e) $\mathrm{cw}$ vortex domain wall transforms to ccw end vortex wall (f) after a field pulse of $7 \mathrm{Oe}$. The ccw wall $(\mathrm{g})$ however does not transform after a field pulse of $11 \mathrm{Oe}(\mathrm{h})$

the mean magnetization is lying parallel to the edge, one of the black lines at the edge is thicker than the other, as the electrons are deflected by the magnetic moments. ${ }^{15}$

In the top wire (sharp corners, geometry 1, Fig. 1) one can see a transverse tail-to-tail ( $\mathrm{t} 2 \mathrm{t}$ ) wall at the $\wedge$-shaped kink and a head-to-head ( $h 2 h)$ vortex wall at the $v$-shaped kink. The vortex wall has clockwise (cw) chirality, seen as a black central vortex core with a black diagonal line connecting the two topological edge defects and two white sidewalls. The vortex walls in geometry 2 have a counter-clockwise (ccw) sense of rotation, with white vortex core, black sidewalls and two topological edge defects (see Fig. 1, arrows). The vortex domain wall in the sharp zigzag wire (geometry 1) however is slightly distorted because the inner diagonal wall rumning through the vortex core, that has opposite contrast compared to the outer area, pins at the inner corner of the kink in order to reduce the stray field energy as the area where spins are pointing perpendicularly to the edge is minimized. ${ }^{16}$ The bottom wire (geometry 3) contains two cw vortex walls (black core). In the case of simple rectangles, end domains can be found because the magnetic moments energetically prefer to lie parallel also to the edges at the end in order to reduce the stray field energy. ${ }^{17,18}$ Depending on the sign of the nucleation field and whether one looks at the left or right end, the end domains in our geometries are also separated by a black or white line corresponding to the spin structure at the end as visualized in Fig. 1. Every wire contains a white line at the left end, looking at the right end of the wire a black line is present. A reversal of the nucleation field (tilt to $-45^{\circ}$ ) creates the opposite contrast for the end domains. The domain walls at the bends also reverse from $h 2 h$ to $t 2 t$ and vice versa [Figs. 2(a) and 2(c). The black line caused by the end domain is at the left, and the white at the right end [Figs. 2(e) and $2(\mathrm{~g})]$.

Next we investigate the magnetic switching. First at small fields of 10.5 Oe the vortex walls in the curved wires start moving. When increasing the pulse height to $15( \pm 5) \mathrm{Oe}$, the transverse walls in the sharp zigzag wires (geometry 1) transform to vortex walls. The vortex walls in the sharp zigzag wires (geometry 1) start moving at $31.5 \mathrm{Oe}$.

When the depinning measurement is repeated for the same kink, we find that the depinning field varies for a given kink by $1-2 \mathrm{Oe}$, whereas from one kink to the next by up to 10 Oe (geometry 1 ) and 3 Oe (geometry 2 and 3 ). Taking the image of a potential landscape, this result suggests that from bend to bend the differences in the local energy minima due

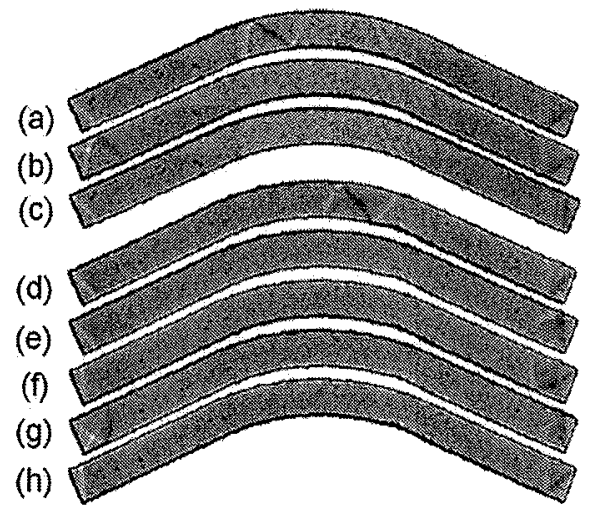

FIG. 3. Two different wires with one bend (width $500 \mathrm{~nm}$ thickness $20 \mathrm{~nm}$ ) (a) Wire 1: $t 2 \mathrm{cw}$ vortex domain wall after nucleation as in Fig. 1. (b) After a magnetic field pulse of 16 Oe an end vortex is formed with opposite (ccw) chirality, the magnetizations points to the right. (c) The end vortex is depinned with a field pulse of $55 \mathrm{Oe}$ and the magnetization switches, the vortex leaves the wire at the right end. (d) Wire 2: $12 \mathrm{cw}$ vortex domain wall after nucleation. (e) Vortex wall moved to the right end and without chirality transformation after a field pulse of $20 \mathrm{Oe}$. (f) The magnetization switches after a field pulse of 70 Oe while the vortex end domain is still pinned. The end domain at the left end changes from "l" to " $p$ " shape. (g) The vortex end domain is depinned at $70 \mathrm{Oe}$ and moves to the left. The chirality transforms to ccw. (h) The end vortex domain is depinned at the left end and leaves the wire at the right after a field pulse of $70 \mathrm{Oe}$.

to structural defects are larger than thermal energy fluctuations at room temperature.

Next we observe the behavior as a vortex wall moves towards the end of the wire and interacts with the end domain to form an end vortex [Figs. 2(b), 2(d), 2(f), and $2(\mathrm{~h})]^{17,19}$ In Fig. 2(a) we can see a cw h2h vortex wall with a black core. After a magnetic field pulse of 31 Oe to the left, the wall moves to the left [Fig. 2(b)] end and stays there. A similar situation is seen in Fig. 2(c) (geometry 2) but the initial vortex wall has a ccw chirality. After magnetic field pulses of 6 Oe the wall moves to the end and the contrast of the core changes to black meaning that the vortex chirality switches from $\mathrm{ccw}$ to $\mathrm{cw}$. This experiment can be repeated and no end vortex with ccw chirality could ever be observed at this end of the wire. When reversing the magnetic field pulse direction and investigating the interaction of a vortex with the spin structure at the right end of the wire the situation is exactly opposite. All the vortex walls with white cores [ccw, Fig. $2(\mathrm{~g})]$, that move to the right end, stay unchanged [Fig. 2(h)], but the vortex walls with black core [cw, Fig. 2(e)] transform to $\mathrm{ccw}$ [white core, Fig. 2(f)]. We can summarize that for the given end domains at the left end only $\mathrm{cw}$ end vortex walls appear (black core) and at the right end only end $\mathrm{ccw}$ vortices (white core) appear regardless of the prior vortex wall chirality.

The same effect is observed with another set of two wires of similar geometry but with only one bend (Fig. 3). The initial magnetization is reversed compared to the previous experiment, as the sample is tilted to $+45^{\circ}$ again for nucleation. So the contrast of the end domain is opposite to the previous case. We also have $t 2 t$ vortex walls, here both with a black core and thus cw chirality, in the bend. When applying magnetic field pulses along the wire direction, the vortex wall moves to the left or right end of the wire depending on the field direction. At the left end the vortex wall transforms to $\mathrm{ccw}$ and at the right the chirality does not change. Thus the chirality of the end vortex configuration is 


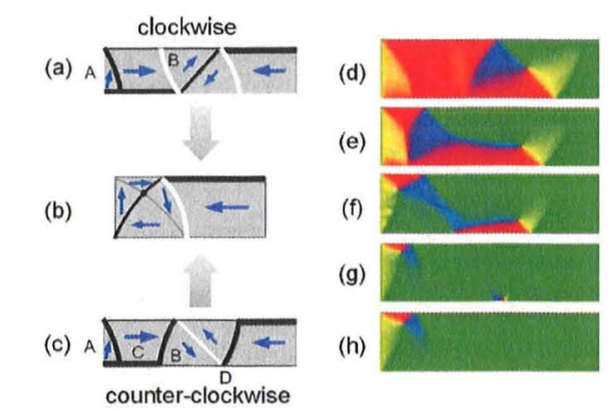

FIG. 4. (Color online) (a) Initial $\mathrm{cw}$ wall: The magnetic moments in $\mathrm{A}$ and $\mathrm{B}$ lie in almost the same direction. Under a field pulse the moments in between can rotate continuously and (b) no chirality transformation visible. (c) Initial ccw wall: The magnetic moments in A and B lie almost antiparallel, the vortex core is expelled in D and a new vortex core is nucleated in $\mathrm{C}$ resulting in (b) a cw end vortex domain. [(e)-(h)] Results of micromagnetic simulation (Ref. 20) (width $500 \mathrm{~nm}, 20 \mathrm{~nm}$ thickness, $2.5 \mu \mathrm{m}$ length, $5 \mathrm{~nm}$ cell size, exchange constant $A=10.05 \times 10^{-12} \mathrm{~J} / \mathrm{m}$, damping constant $\alpha=0.1$, and the saturation magnetization $M_{s}=800 \times 10^{3} \mathrm{~A} / \mathrm{m}$ ). (d) Initial state. (e) Magnetic field of 24 Oe applied, the left half of the vortex wall interacts with the end domain, while the vortex core moves toward the bottom edge of the wire. (f) A new vortex core is nucleated in the area where both walls meet (between blue and yellow). (g) The old vortex core is pushed further to the bottom edge and it is more or less separated from the end vortex domain. (h) Final result which is the same as in our experiments.

opposite when the nucleation field is reversed. Even if the magnetization in the wire is pointing from left to right [see Fig. 3(f)] as in the previous experiment, it is the spin structure at the end that determines the chirality of the end vortex [see Fig. 3(g), ccw]. Comparing Figs. 3(f) and 3(g) and Figs. 2(c) and 2(d) we see that it is the spin structure at the end that sets the end vortex chirality, since for the same field and vortex wall motion direction the two different end domains result in two different end vortices.

There are two cases to be explained: the nontransformation case where the initial vortex domain wall has already the final chirality. Here the magnetic moments in the initial end domain, marked in Fig. 4(a) with A, and in the end vortex domain at $\mathrm{B}$ almost point to the same direction. When the wall meets the end domain the magnetic moments between $\mathrm{A}$ and $\mathrm{B}$ can rotate continuously.

The case of the transformation is more complicated. When the vortex domain wall moves close to the end domain, two areas A and B with almost antiparallel magnetization meet [Fig. 4(c)]. If the end domain wall is pinned due to imperfections at the wire edges, the vortex cannot move further without being transformed. In order to understand the interaction of vortex domain wall and end domain better, micromagnetic simulations were carried out. ${ }^{20}$ The case of nontransformation is reproduced by applying a small magnetic field to a rectangular wire. The vortex wall moves toward the end of the wire. When vortex wall and end domain meet, they form the end vortex with the moments in between rotating continuously.

Micromagnetic simulations reproduce the chirality transformation behavior if the spins at the end of the wire are pinned $(1 \mathrm{kOe})$. This pinning accounts for the edge roughness and possible defects in the real sample. The effect of such a pinning is seen in the experiment, where we observe that only with increasing the field above 50 Oe or more the end vortex is expelled, whereas without the pinning the end vortex is expelled in the simulation already at 20 Oe. After applying a 40 Oe field, a transformation of the vortex is found with the same result as in the experiments [see Fig. 4(h)]. The transformation itself is predicted to be rather complex: The outer left half of the initial vortex wall interacts with the end domain [Fig. 4(e)] and a new vortex core is nucleated in this area [Fig. 4(f)]. The old vortex core moves toward the bottom edge of the wire [Figs. $4(\mathrm{e})-4(\mathrm{~g})]$ and is finally expelled [Fig. 4(h)]. One initially expects that the nucleation of the new core could be influenced by defects in a real wire, however we observe this rectification in at least seven different wires with four different geometries. This means that the effect is rather robust and allows for reliable rectification of the wall chirality.

In conclusion, we have determined the interaction between a vortex wall and an end domain and a very stable end vortex spin configuration with a chirality that depends only on the direction of spins in the prior end domain rather than on chirality of the incoming vortex is found. With a controlled pinning site for the end domain and a reproducible depinning behavior of the end vortex this constitutes a "chirality rectifier" for magnetic logic and other devices.

We acknowledge support by the DFG (KL 1811 and SFB767), the EU (RTN SPINSWITCH MRTN-CT-2006035327, Stg MASPIC ERC-2007-Stg 208162) and the Samsung Advanced Institute of Technology.

'S. S. P. Parkin, M. Hayashi, and L. Thomas, Science 320, 190 (2008).

${ }^{2}$ D. A. Allwood, G. Xiong, C. C. Faulkner, D. Atkinson, and D. Petit, Science 309, 1688 (2005).

${ }^{3}$ M. Hayashi, L. Thomas, C. Rettner, R. Moriya, and S. S. P. Parkin, Nat. Phys. 3, 21 (2007).

${ }^{4}$ M. Kläui, J. Phys.: Condens. Matter 20, 313001 (2008).

${ }^{5}$ R. D. McMichael and M. J. Donahue, IEEE Trans. Magn. 33, 4167 (1997).

${ }^{6}$ D. McGrouther, S. McVitie, J. N. Chapman, and A. Gentils, Appl. Phys. Lett. 91, 022506 (2007).

${ }^{7}$ E. Feldtkeller and H. Thomas, Phys. Kondens. Mater. 4, 8 (1965).

${ }^{8}$ J. He, Z. Li, and S. Zhang, Phys. Rev. B 73, 184408 (2006).

${ }^{9}$ D. Petit, A.-V. Jausovec, D. Read, and R. P. Cowburn, J. Appl. Phys. 103, 114307 (2008).

${ }^{10}$ M. Hayashi, L. Thomas, C. Rettner, R. Moriya, X. Jiang, and S. S. P. Parkin, Phys. Rev. Lett. 97, 207205 (2006).

${ }^{11}$ L. K. Bogart, D. Atkinson, K. O'Shea, D. McGrouther, and S. McVitie, Phys. Rev, B 79, 054414 (2009).

${ }^{12}$ M. Kläui, P.-O. Jubert, R. Allenspach, A. Bischof, J. A. C. Bland, G. Faini, U. Rüdiger, C. A. F. Vaz, L. Vila, and C. Vouille, Phys. Rev. Lett. 95 026601 (2005).

${ }^{13}$ C. K. Lim, G. Yi, J. N. Chapman, W. A. P. Nicholson, S. McVitie, and C. D. W. Wilkinson, J. Phys. D: Appl. Phys. 36, 3099 (2003).

${ }^{14}$ J. N. Chapman, J. Phys. D: Appl. Phys. 17, 623 (1984).

${ }^{15}$ K. J. O'Shea, S. McVitie, J. N. Chapman, and J. M. R. Weaver, Appl. Phys. Lett. 93, 202505 (2008).

${ }^{16}$ M. Kläui, H. Ehrke, U. Rüdiger, T. Kasama, R. E. Dunin-Borkowsky, D. Backes, L. J. Heyderman, C. A. F. Vaz, J. A. C. Bland, G. Faini, E. Cambril, and W. Wernsdorfer, Appl. Phys. Lett. 87, 102509 (2005).

${ }^{17}$ K. J. Kirk, Contemp. Phys. 41, 61 (2000).

${ }^{18}$ R. E. Dunin-Borkowski, M. R. McCartney, B. Kardynal, S. S. P. Parkin, M. R. Scheinfein, and D. J. Smith, J. Microsc. 200, 187 (2000).

${ }^{19}$ J. Shi, S. Tehrani, T. Zhu, Y. F. Zheng, and J.-G. Zhu, Appl. Phys. Lett. 74, 2525 (1999).

${ }^{20}$ LLG (http://llgmicro.home.mindspring.com). 